\title{
Polymyxin B hemoperfusion in acute aortic thrombosis secondary to salmonellosis: a case report
}

\section{$\underline{\text { R. Arranz Pérez }}{ }^{1}$, J. Montoro Garcia², E. Terradillos Martin ${ }^{1}$, I. Frias Soriano ${ }^{1}$}

${ }^{1}$ Department of Anaesthesia Hospital General Universitario Gregorio Marañon - Madrid (Spain), ${ }^{2}$ Department of Anaesthesia Hospital General Universitario de Guadalajara - Madrid (Spain).

\section{BACKGROUND:}

We present the case of a complete infrarenal abdominal aorta thrombosis secondary to an infectious aneurysm due to sepsis by Samonella of group D with symptoms of acute lower limb ischemia and paraplegia, with a favorable evolution after hemoperfusion with polymyxin B (PMX-B), once it was intervened urgently.

\begin{abstract}
CASE REPORT:
A 57-year-old male with COPD, debuted with a paresthesia in the lower limbs that leads to paraplegia 48 hours in the context of gastroenteritis and fever related to egg ingestion, before his admission to the emergency department (ED). In the ED, our patient had dehydration signs, pallor and pulselessness in lower limbs. An urgent CT scan reveals an aneurysm and complete thrombosis of the infrarenal abdominal aorta (Image 1), which is why he was immediatly transferred to the operating room where axillo-bifemoral bypass is performed. He developed a reperfusion syndrome, multiorganic dysfunction and a septic shock that required vasoactive support and hemodiafiltration, including two cycles of B polymyxin in addition to intravenous ceftriaxone when isolating on the 2 nd day of samonella from group $D$ in the blood cultures. The treatment led to a clinical improvement of the patient with recovery and he was discharged in a rehabilitation program.
\end{abstract}

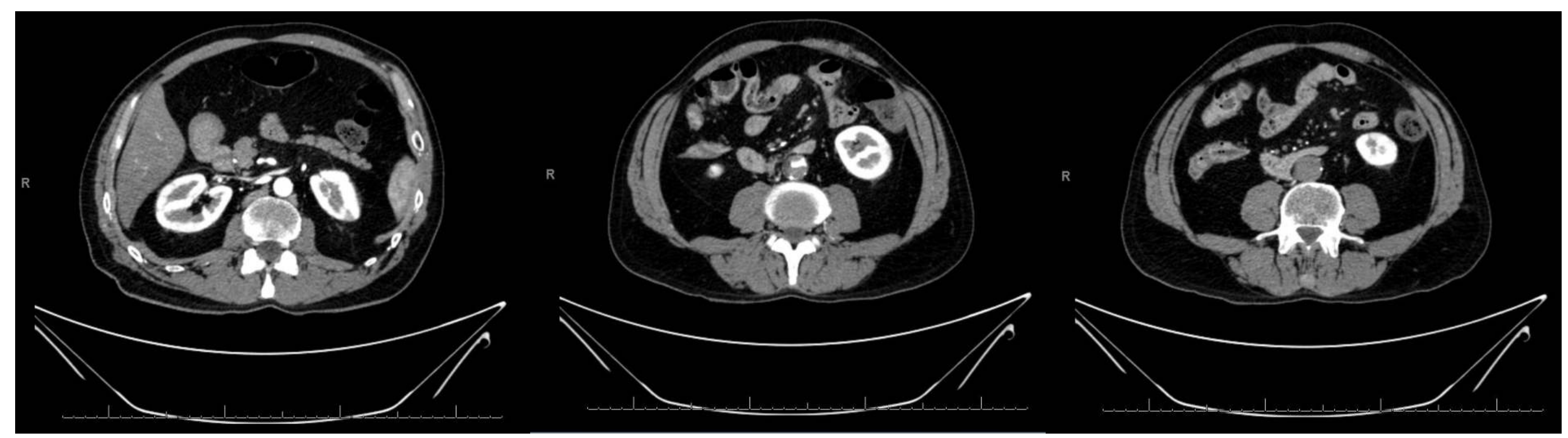

Image 1: CT scan of infrarenal abdominal aortic aneurysm and complete thrombosis, with no evidence of contrast in the lumen.

\section{DISCUSSION:}

The aortic occlusion syndrome is a rare entity with high morbidity and mortality. Infectious origin aneurysm are among its causes, and represent less than $2 \%^{1}$. The clinical manifestations are variable and are characterized by symptoms of acute arterial ischemia in the lower limbs, as well as symptomatic neurological deficit, acute abdomen or renal failure. The location and proximity of the thrombus within the aorta determines the clinic. Salmonella spp sepsis occasionally produces aneurysms in addition to states of systemic hypercoagulability, which facilitates vasculature thrombosis formation. Early diagnosis is indispensable and the TC is technique of choice. Urgent intervention is obligatory through endovascular techniques or bypass surgery. PMX-B hemofiltration in addition to standard treatment in cases of severe septic shock, seems to decrease mortality ${ }^{2}$.

\section{LEARNING POINTS:}

Acute aortic thrombosis of infectious origin is a rare entity with a high morbimortality that requires early diagnosis and treatment. The formation of thrombi and aneurysm could be associated with Salmonellosis. B polymyxin hemofiltration could contribute to improve survival in gram negative bacteria sepsis shock.

1. Jiménez C, Valencia Á, Jaramillo C, Correa JR. Bacterial aortic aneurym due Salmonella spp. infection. RevColomb Cir. 2011;26:214-221.

2. Terayama T et al. Polymyxin B Hemoperfusion for Sepsis and Septic Shock: A Systematic Review and Meta-Analysis. Surg Infect (Larchmt). 2017 Apr;18(3):225-233 